\title{
General Overview of "Human Dementia Diseases": Definitions, Classifications, Neurobiological Profiles and Clinical Treatments
}

ISSN: 2578-0093

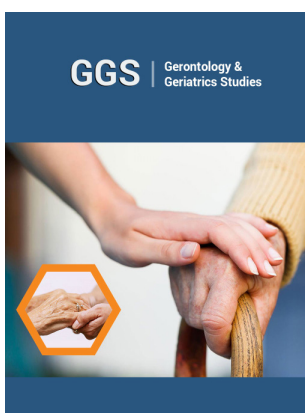

*Corresponding author: Giulio Perrotta, Department of Psychology, Italy

Submission: 侮 March 13, 2020

Published: 剕May 07, 2020

Volume 6 - Issue 1

How to cite this article: Giulio Perrotta. General Overview of "Human Dementia Diseases": Definitions, Classifications, Neurobiological Profiles and Clinical Treatments. Gerontol \& Geriatric stud. 6(1). GGS.000626. 2020.

DOI: $10.31031 /$ GGS.2020.06.000626

Copyright@ Giulio Perrotta, This article is distributed under the terms of the Creative Commons Attribution 4.0 International License, which permits unrestricted use and redistribution provided that the original author and source are credited.

\section{Giulio Perrotta*}

Department of Psychology, Italy

\begin{abstract}
This work focuses on the specific analysis of the general, neurobiological and clinical profiles of human dementias, starting from classifications and definitions, and then extending the field of study to clinical and psychological symptomatologic data, to conclude with treatments and better management of these patients.
\end{abstract}

\section{Introduction}

Definitions and classifications [1] "Dementia” is a global, chronic and irreversible cognitive deterioration, even if with a correct diagnosis and targeted therapy it is possible to slow down the progression of the disease. The diagnosis is therefore clinical, through laboratory tests and the study of MRI images, starting from the symptoms described in the anamnestic history. The treatment, therefore, is supportive, precisely by virtue of the chronic, progressive, disabling and irreversible nature of this family of pathologies. Dementia can occur at any age but mainly affects after the fifth decade of life. Based on the data found in the anamnesis, dementias can be classified into:

A. "Corticals" or "Subcorticals". The "cortical" are so defined because they mainly affect the structures of the cerebral cortex and are characterized by deteriorations in the cognitive, memory and language levels, have extensive cortical atrophy, early alterations of memory, loss of abstract thought, agnosia, aphasia and apraxia. The following belong to these categories: Alzheimer's disease, Creutzfeld-Jacob disease and Pick's disease. The "subcorticals" are dementias that mainly affect structures that are located below the cerebral cortex and are characterized by deteriorations in the personality, emotional sphere and motor functions; they present a slowdown in motor responses (called bradyphrenia), personality alterations typical of affective disorders such as apathy and depression, less memory loss and absence of disorders considered "cortical" such as agnosia, aphasia, apraxia. These categories include Parkinson's disease, Huntington's chorea, Progressive supranuclear palsy and Vascular dementias (due to areas of cerebral necrosis caused by a deficiency in the blood circulation). The two classifications described above, however, are generally considered too rigid, in light of recent acquisitions in the neuropsychological and anatomical-physiological fields, and are not accepted by all experts.

B. "Irreversible" or "Reversible". Instead, based on an etiopathogenetic classification, dementias can be distinguished in irreversible and reversible forms. The latter category includes secondary dementia forms, that is, those caused by a primary factor that led to dementia. These include the deficiencies of vitamin B12, folate and thyroid, liver and kidney diseases. Furthermore, brain infections, neoplasms and normotensive hydrocephalus (which presents itself with the symptomatic triad of walking disorders, urinary incontinence and cognitive decline). A separate discussion must be made for Wernicke-Korsakoff syndrome, which falls within the irreversible forms although it depends on a deficiency of thiamine. The latter form is a degeneration of the central 
nervous system affecting the frontal regions, the hippocampus, the mammillary bodies and the mid-dorsal nucleus of the thalamus; the therapy is moisturizing but no improvement is possible, since neuronal degeneration determines a clinical picture characterized by anterograde amnesia, a decrease in learning ability and confabulation (that is, patients fill their memory lapses with delusional fantastic productions). All the other forms obviously fall into the category of irreversible pathologies.

C. "Common" or "rare": based on the incidence rate in the population.

D. In the medical clinic it is preferred to classify them according to the following scheme:

1) Pathology determined by beta-amyloid deposits and neurofibrillary tangles: Alzheimer's.

2) Pathology determined by Tau protein anomalies: Chronic traumatic encephalopathy, Corticobasal degeneration, Frontotemporal dementia, among which Pick disease, Progressive primary aphasia and Semantic dementia-, and Progressive supranuclear paralysis.

3) Pathology determined by alpha-synuclein anomalies: Lewy and Parkinson's body dementia.

4) Pathology determined by the mutation of the huntingtin's gene: Huntington.

5) Pathology determined by celebrovascular damage: Binswanger disease, Lacunar syndrome, Multi-infarct dementia, Dementia from a single strategic heart attack and Cerebral hypoxia.

6) Pathology determined by ingestion of toxic or pharmacological substances: alcoholism and exposure to heavy metals.

7) Pathology determined by infectious agents: fungal forms (cryptococcosis dementia), bacterial forms such as spirochetes (syphilis and Lyme) and viral forms (post-encephalitic Herpes syndromes or forms associated with HIV).

8) Pathology determined by prion glycoprotein: GerstmannSträussler-Scheinker, Fatal familial insomnia, Creutzfeldt-Jakob and its western variant "nvMCJ" and aboriginal "Kuru".

9) Pathology determined by disorders of the brain structure: external trauma, tumors, normal tension hydrocephalus.

10) Pathology determined by a reversible condition: thyroiditis, liver disease, pancreatitis, chronic kidney disorders, vitamin and amino acid deficiencies.

However, "dementia" should not be confused with other more or less pathological forms:

a) "delirium": dementia mainly affects memory and is generally caused by structural changes in the brain, has a slower onset, and is generally irreversible; delirium mainly affects attention, it is generally caused by an acute disease or drug toxicity (sometimes life-threatening) and is often reversible (if a patient is inattentive, the most likely diagnosis is delirium, however in advanced stages of dementia can also be severely impaired by attention).

b) "amyotrophic lateral sclerosis" (or Lou Gehrig's disease, Charcot's disease, motor neuron disease) is a progressive neurodegenerative motor neuron disease, which selectively affects motor neurons, both central (" 1 st motor neuron", at the level of the cerebral cortex), and peripheral ("2nd motor neuron", at the level of the brain stem and spinal cord). It is characterized by muscle stiffness, muscle contractions and gradual weakness due to the decrease in muscle size. This results in difficulty speaking, swallowing and finally breathing. Although it shares some typical symptoms of dementia, it is not considered a real dementia form.

c) "multiple sclerosis", which is a demyelinating neurodegenerative disease, that is, with lesions affecting the central nervous system. For many years it has been considered a white matter disease of the central nervous system, however an increasing number of studies have also shown an involvement of the gray matter. In multiple sclerosis, myelin damage and loss occurs in multiple areas (hence the name "multiple") of the central nervous system. Numerous clinical and experimental evidences indicate that the reaction of the immune system is at the basis of the disease which triggers an attack against myelin This attack consists of an inflammatory process that affects limited areas of the central nervous system and causes the destruction of myelin and cells specialized, the oligo-dendrocytes, which produce it. These areas of myelin loss (or "demyelination"), also called "plaques", can be scattered anywhere in the cerebral hemispheres, with a preference for optic nerves, cerebellum and spinal cord. At the base therefore there is a process of demyelination that determines damage or loss of myelin and the formation of lesions (plaques) that can evolve from an initial inflammatory phase to a chronic phase, in which they take on characteristics similar to scars, from which the term derives "sclerosis".

d) "age-associated memory impairment": memory impairment does not affect daily functioning. If these subjects are given enough time to learn new information, their intellectual performance is good.

e) "mild cognitive impairment": memory and / or other cognitive functions are impaired, but the disorder is not severe enough to interfere with daily activities.

f) "cognitive symptoms related to depression": these cognitive disorders are resolved with the treatment of depression. Elderly depressed patients may experience cognitive decline, but unlike dementia patients, they tend to exaggerate their memory loss and rarely forget important or personal events. Neurological examination is normal except for signs of psychomotor slowdown. When evaluated with tests, patients with depression make little effort to respond, while 
those with dementia try hard even if they respond incorrectly. When depression and dementia coexist, the treatment of depression is not able to completely regress the cognitive disorder.

\section{Symptomatologic Profiles}

\section{Neurophysiological symptoms}

In detail, the "main dementia forms":

Alzheimer's [2]: It is the most common chronic progressive and disabling degenerative neurocognitive dementia (between $50 \%$ and 70\%), closely connected to deposits of amyloid plaques and neurofibrillary tangles, capable of causing the following symptoms, divided into four specific phases: 1) pre-dementia (short-term memory deficit and inability to acquire new information; 2) initial phase (language deficit such as impoverishment in vocabulary and decrease in fluency of speech, agnosia, apraxia, decreased coordination ability muscle, with less damage in episodic and implicit memory); 3) intermediate phase (aphasia, disorientation, inability to deal with daily activities, long-term memory deficit, sensory dysfunction, mood swings, depression); 4) final phase (total dependence on the caregiver, muscle weakness, inability to feed independently, death from infection or neurovascular damage). The prognosis is 3-9 years.

Chronic traumatic encephalopathy [3]: Also known as boxer dementia, it is a dementia caused by anomalies of the Tau protein, following repeated head injuries and concussions, capable of interfering with the normal metabolism of neurons. Symptoms that distinguish the syndrome include tremors, ataxia, dysarthria and pyramidal lesions, also alteration of the personality, with consequent outbursts of anger, delirium, and obsessive jealousy. The prognosis is $2-5$ years.

Frontotemporal dementias [4]: Also called in DSM-5 with the term "major or mild frontotemporal neurocognitive disorder", this phrase identifies a heterogeneous group of non-Alzheimer's neurodegenerative dementias that are characterized by the presence of degenerative-atrophic alterations of the frontal and temporal brain lobes. It is an umbrella term for different pathologies or alternatively a pathology with different variants. Symptoms such as sudden personality changes and behavioral-motor oddities in the absence of pre-existing psychiatric or neurological pathologies, and only afterwards for a partial memory loss, together with the growing cognitive and motor deficit, are reported. Average survival is approximately 6-8 years, based on the specific form and timing of intervention. Typical forms are:

a) Corticobasal degeneration. It is a rare neurodegenerative disease, frontotemporal and characterized by dysfunctions of the motor system and multiple cognitive deficits, such as asymmetric rigidity, bradykinesia, limb apraxia and visualspatial deficit. CBS has wide clinical variability among patients, many of whom develop a relatively pure motor syndrome, while others have an association of motor and cognitive deficits. The onset of the disease is insidious and usually occurs in the sixth or seventh decade of life, with signs that initially tend to be unilateral, with greater involvement of the arms than the legs. The disease mainly begins in the form of movement anomalies, with stiffness, bradykinesia and tremors, associated with frontal signs, loss of cortical sensitivity, alien limb phenomenon, stimulated induced myoclonus and progressive limb apraxia, which with the progression of the disease they can become bilateral, although they are usually asymmetric. Gait abnormalities and postural instability are common, while dystonia and aphasia (apraxia of speech, dysfluency and geography) are less common. Eye movement abnormalities and behavioral disturbances may also be present. In the later stages of the disease, many patients have dementia. Differential diagnosis includes progressive non-fluent aphasia and, to a lesser extent, behavioral variant of front-temporal dementia, progressive supranuclear palsy, multiple systemic atrophy, posterior cortical atrophy, Alzheimer's disease (in the typical/ amnesic form) and idiopathic Parkinson's disease. The prognosis is 5-8 years.

b) Pick disease. It is a rare degenerative frontotemporal form, characterized by dementia but unlike the subject with pure dementia, Pick's patient loses his expressive-expository skills much more quickly, but not those of reading and writing, which are instead preserved more long over time although difficult. The anatomopathological picture is characterized by atrophy of the front-temporal area and by the presence of characteristic neuronal alterations constituted by Pick's bodies, which are filamentous intracellular inclusions composed of neurofilaments, similar to the inclusions observed in Alzheimer's disease. It therefore affects earlier than Alzheimer's. The prognosis is 5-7 years on average and varies from 2 years in aggressive forms to $12-15$ years in light forms or supported by adequate therapy.

c) Frontotemporal dementia associated with parkinsonism of Chromosome 17 or FTDP-17. It is an autosomal dominant neurodegenerative disease with complete penetrance that manifests itself with progressive dementia that occurs between the third and fourth decade of life and that is associated with Parkinson-like akinetic-hypertonic manifestations. As suggested by the name, FTDP-17 is due to a mutation of the gene encoding the Tau protein, present on chromosome 17. This mutation, most frequently localized in the portion coding for the binding site between the tau protein and tubulin, leads to anomalies of the neuronal cytoskeleton due to impediment of the formation of microtubules. In a lesser percentage of cases, the mutation is localized in a non-coding region that regulates the transcription of exon 10 of the tau protein; in this case, the mutation results in the production of a longer protein, tending to neuronal accumulation and consequent cytotoxicity due to altered disposal. Although defined as frontotemporal dementia (FTD), FTDP-17 differs from this heterogeneous group of diseases due to the earliness of the manifestations (third-fourth decade of life) and the presence of precipitous alterations of social conduct often absent from FTD and preceding years of cognitive and motor disorders. Subsequently, language deficits 
with fluent aphasia and disturbances of the extrapyramidal system (Parkinson-like picture) characterized by dystonic and / or myoclonus phenomena appear. Typical in the fullblown phase is the onset of a pseudobulbar syndrome, with dysphagia and changes in ocular motility. Cerebral atrophy is more pronounced in the mesencephalic, pontine and frontal, temporal and parietal lobes. The prognosis is 5-10 years.

d) Frontotemporal dementia with amyotrophy. The definition of frontotemporal dementia with amyotrophy collects the nosologically classification criteria of both dementias and motor neuron diseases. Dementia occurs early compared to motor impairment; it is characterized by the presence of persevering and stereotyped attitudes, dysphasia, disinterest in personal care, disinhibition and hypersexuality in the absence of memory disorders. Motor impairment, often late, is instead part of amyotrophic lateral sclerosis; in this sense, frontotemporal dementia with amyotrophy is characterized by the presence of atrophic changes in the first and second motor neurons. The alterations of personality and language typically arise in the presenile period, with a short duration of illness, generally 2-3 years, with a result towards respiratory failure. Typical is the rarefaction of the neurons of the frontal and temporal lobes and the anterior horns of the spinal cord.

Primary progressive aphasia [5]: It is a progressive speech disorder that, although it is part of the frontotemporal dementia, differs from semantic dementia in the most severe aphasia and in the absence of personality, behavior and anosognosia disorders, although dementia invariably occurs in late stages of illness. The prognosis is 8-15 years.

Semantic dementia [6]: Unlike primary progressive aphasia, aphasia is moderate in this pathology where fluency or the ability to find words is not corrupted. Rather, there is a progressive loss of the meaning of words, very often associated with paraphasia's and prosopagnosia. The disinterest in personal care, persevering attitudes and compulsiveness, identify this nosologically entity in the context of frontotemporal dementia. This link is strengthened by the presence of impaired mnemonic functions only in late stages of the disease. The prognosis is 8-15 years.

Progressive supranuclear palsy [7]: Neurodegeneration involves atrophy at the level of the midbrain and other brain structures including the subthalamic nucleus, the Globus pallidus, the nuclei of the bridge and the black substance. The name of the disease describes the supranuclear localization (with reference to the oculomotor nuclei of the midbrain) of pathological changes. The term 'nucleus' refers to the group of neurons from which the oculomotor cranial nerves that connect the midbrain and extrinsic muscles of the eye originate. While a paralysis of the oculomotor nerves can be caused by lesions of the nerves themselves or by lesions at the level of the nuclei, in the case of supranuclear palsy the cause is located in the brain, above those structures. The onset can be non-specific, with an often symmetrical and rigid-bradykinetic extrapyramidal syndrome, but in $2 / 3$ of the cases there is a disturbance of the balance with a tendency to retropulsion, postural instability and frequent falls. More rarely the onset occurs with ophthalmoplegia and other truncal signs. In the more advanced stages of the disease, the appearance of the typical supranuclear ophthalmoplegia makes the clinical picture more characteristic: there is paresis of the gaze vertically (both downwards and upwards). In the late stages, voluntary eye movements are missing, but ocular-vestibular reflexes are discreetly preserved (thus giving rise to the so-called doll-eye phenomenon: the gaze is immobile even when the head is turned passively, the eyes always point in the same direction - therefore a automatic movement of the eyeballs stimulated by the vestibular system, while voluntary control of eye movement is no longer possible). Blinking rarities and oculo-eyelid apraxia may also occur. All this gives the patient an astonished appearance with the need to move the head to look around. The movements of the head are also reduced, however, as muscle stiffness is often particularly accentuated at the level of the trunk and cervical musculature. Thus, instead of the flexion posture typical of parkinsonians, on the contrary in PSP patients there is an attitude in extension, with stiff neck. Other signs are of the "pseudobulbar" type: dysarthria, dysphonia, dysphagia, with laughter and spastic crying (involuntary, not necessarily corresponding to the respective emotional states). There may be vivid or increased reflexes with bilateral Babinski phenomenon. Also common are signs of cognitive impairment, sometimes blepharospasm is present. Especially in the early stages of the disease, PSP can be difficult to distinguish from Parkinson's disease. The most important differences that allow to make the differential diagnosis are: both paralysis and Parkinson's disease give: rigidity, difficulty of movement, and clumsiness; generally, people with paralysis tend to tilt their heads backwards (and tend to fall backwards), while those with Parkinson's disease usually bend forward (interspersion); speech and swallowing disorders are much more common and severe in paralysis than in Parkinson's disease and also tend to occur earlier; eye movements are abnormal in paralysis, but are almost normal in Parkinson's disease; tremor is more characteristic for Parkinson's disease, while it is rarer in paralysis; people with Parkinson's disease have significant benefits with levodopa, instead people with paralysis do not respond to this drug or respond only transiently. The prognosis is 6-10 years.

Lewy body dementia [8]: It is a neurodegenerative disease, a form of dementia similar to Alzheimer's disease, but with an earlier onset. It has close, but not completely clarified, relationships with Parkinson's disease, with which it shares some anatomopathological aspects: in fact, Lewy bodies are defined as anomalous deposits of fibrillary protein that form in nerve cells and are mainly located in the nuclei of the brain stem (Parkinson's disease) and cerebral cortex (Lewy body dementia). This disease also has some clinical aspects in common with Parkinson's, such as tremors and movement disorders, and it is now believed that the forms of Parkinson's disease associated with (or evolved in) dementia are nothing but Lewy body dementias start with parkinsonism. The symptomatology, although very varied, is characterized by large variations in the state of attention and alertness, with fluctuations present day by day and even hour by 
hour, cognitive disturbances, recurrent visual hallucinations (minor in typical Parkinsonian dementia), and characteristics motor skills. From a clinical point of view, the salient aspects of the disease are: cognitive impairment (with memory problems, semantic dementia and apraxia), characteristically fluctuating (not stable and progressive as in Alzheimer's, at least in the early stages), visual hallucinations, which often mark the onset of the disease, mood disorders (especially depression) and other neuropsychiatric symptoms (delirium, paranoia, anxiety, panic, not showing instead the typical disinhibition of frontotemporal dementias, even if it can be present just like in Parkinson's, nor hyperorality) and then the typical movement disorders which, indistinguishable from those of Parkinson's: tremor at rest, only sometimes kinetic, camptocormia (flexed forward position), Gowers trait (parkinsonian gait), rigidity, amimia, dysarthria, shuffling step; they are often more pronounced and disabling than in Parkinson's, but appear later. Patients are often also subject to hyper catabolism which, if left untreated, can lead to sudden and drastic weight loss. Sleep disturbances (dyssomnia and parasomnias) are frequent, both insomnia and hypersomnia: many patients have sleep behavior problems, manifesting physical reactions, sometimes becoming dangerous for themselves and others. The autonomic nervous system can dysregulate internal functions (dysautonomia), such as blood pressure and temperature: fainting, hyperhidrosis (excessive sweating) and anhidrosis (absence of sweating), xerostomia (dry mouth), incontinence or urinary retention may occur and constipation. The prognosis is 5-8 years.

Parkinson [9]: It is a neurodegenerative disease. The typical motor symptoms of the condition are the result of the death of cells that synthesize and release dopamine. These cells are found in the substantia nigral, a region of the midbrain. The disease is characterized by the accumulation of a protein, called alphasynuclein, in inclusions called Lewy bodies in neurons and by the insufficient formation of dopamine. The anatomical distribution of Lewy bodies is often directly related to the expression and degree of clinical symptoms of each individual. At the onset of the disease, the most obvious symptoms are related to movement, and include tremors, stiffness, slow movements and difficulty walking. Later, cognitive and behavioral problems can arise, with dementia sometimes occurring in the advanced stages. The prognosis is 5-15 years, but it depends on the age of the onset of the first symptoms.

Huntington [10]: It is a neurodegenerative genetic disease that affects muscle coordination and leads to cognitive decline and psychiatric problems. It typically begins during middle age; it is the most frequent disease due to genetics in neurological clinical pictures with abnormal involuntary movements (which are called chorea). The symptoms of the disease can vary between individuals and even among affected members of the same family, but their progression can usually be predicted. The first symptoms are often subtle mood or cognitive problems followed by a general lack of coordination and an unstable gait. As the disease progresses, uncoordinated movements of the body become increasingly evident and are accompanied by a decrease in mental abilities and behavioral and psychiatric problems. Complications, such as pneumonia, heart disease and physical damage from falls, reduce life expectancy to around 10-15 years, starting with the onset of the first symptoms.

Binswanger disease [11]: Also called subcortical vascular dementia, it is a form of dementia, particularly leukoencephalopathy. The most common feature of this disease is a psychomotor slowness. This is caused by damage in deep layers of the white matter of the brain and by arteriosclerosis, as well as by different types of gliosis (microgliosis and astrocytic gliosis), causing the atrophy of the white matter itself and the rupture of the subcortical neural networks, which control the executive cognitive functioning. It is a progressive degenerative disease that rapidly advances with sudden and gradual changes. The main symptoms are directly related to the breakdown of the neural networks. They include short-term memory loss, short-term attention, inadequate behavior, inability to make organizational and decisions, changes in mood and personality, and unstable gait (accompanied by frequent falls). In general, a common psychomotor slowness prevails. Other symptoms are: speech changes and urinary symptoms (unrelated to urological diseases). Life expectancy is 5-8 years.

Lacunar syndrome [12,13]: The anatomopathological term "lacuna" indicates a cavity filled with cerebrospinal fluid, less than $15 \mathrm{~mm}$, located in the gray nuclei, in the thalamus, in the semi-oval center or in the brainstem. Three types have been described: lacunar infarcts, lacunar hemorrhages and dilations of the perivascular spaces of Virchow-Robin. Most of the gaps are silent, but various clinical pictures can be found. The more specific ones are called "lacunar syndromes": pure motor hemiparesis, pure sensory hemiparetic, sensory-motor hemiparesis and ataxic hemiparesis. The diagnosis of lacunar infarction is faced with a lacunar syndrome associated with diagnostic data for images compatible with the occlusion of a single perforating artery, in the absence of another identified etiology. The most frequent cause of lacunar infarction is lipoylations of the perforating arteries, generally favored by chronic arterial hypertension. Lacunar infarcts account for a quarter of cerebral infarcts. The treatment of the acute phase is identical to that of the other cerebral infarcts. Secondary prevention includes antihypertensives, statins and platelet aggregation inhibitors. This subtype of cerebral infarction exposes to relapses and cognitive disturbances. The prognosis depends on the timing of surgery.

Vascular dementias $[\mathbf{1 4 , 1 5 ] : ~ T h e y ~ a r e ~ c o g n i t i v e ~ d e f i c i t ~ c a u s e d ~}$ by an altered blood circulation in the brain, which deprives some areas of the organ of blood, causing the progressive and irreversible death of brain cells. The causes that can distort the normal blood vessel system inside the brain are different: the main one is the socalled small blood vessel disease (subcortical vascular dementia), but less common causes, such as stroke, should not be overlooked mixed dementia, multi-infarct dementia, CADASIL, hypoxic encephalopathy and atherosclerosis. The symptoms of vascular dementia can be very different from patient to patient, depending on the parts of the brain affected by the disease. The prognosis here too is highly variable but hardly exceeds the first decade of diagnosis. 
Gerstmann-Sträussler-Scheinker disease [16]: It is a very rare neurodegenerative disease (1-3 cases per 100 million) and of a purely family character with autosomal dominant inheritance, classified as transmissible spongiform encephalopathy. It is one of the few diseases that are caused by prions, a class of highly protease-resistant pathogenic mutated proteins. In the classic form, the mutation of the PRP protein occurs at the level of codon 102. In others, mutations at the level of codons 198,217 and 117 hesitate towards a form dominated by dementia and parkinsonism. Furthermore, a codon 105 mutation characterized by late dementia and spastic paraparesis is recognized. Symptoms develop slowly in the initial phase, with difficulty articulating words (dysarthria), progressive cerebellar ataxia and pyramidal signs, with postural instability and impaired motor function. Subsequently, the disease progresses with the accentuation of dementia. The prognosis hardly exceeds 5 years.

Fatal family insomnia [17-19]: It is an autosomal dominant prion genetic disease that leads to degeneration of the nucleus of the thalamus and cerebral cortex. The mutated PRNP gene encodes the PrPSc protein, which tends to fold in an incorrect way, called a prion form. Familial fatal insomnia is a degenerative brain disease belonging to the group of spongiform encephalopathies. It causes total insomnia (inability to sleep), which after a few months of suffering leads to death. It appears that the mechanism of disease onset is linked to hormonal changes, including an increase in catecholamines and cortisol, a lack of melatonin and a loss of the circadian rhythm of the pituitary gland. Furthermore, according to some scientific studies, the PrPc protein is a component of GABA receptors and its conversion into a protein isoform would cause the inability of the same receptors to exert their inhibitory action on the nerve cells of the thalamus, which, due to the 'excessive activation, would face necrosis. After a period of exhaustion, the patient begins to suffer from increasingly severe drug-resistant insomnia, up to the complete inability to sleep, which is followed by stupor and coma. However, in the stage where the inability to sleep occurs, there are apparently paradoxical sleep phases similar to sleepwalking, which are characterized by recited dreams. The disease progressively damages verbal and long-term memory but does not involve dementia. In addition to insomnia, motor difficulties (e.g. ataxic gait) and an early neurovegetative dysautonomia, with sphincter disorders, sialorrhea, watery eyes, constant sweating, runny nose, increased body temperature and blood pressure, tachycardia and changes in breathing (e.g. tachypnea), but sometimes paresis also appears. The prognosis is always unfavorable: the duration before death varies from 6 to 18 months, but on average it is around 12 months.

Creutzfeldt-Jakob and its western variant "nvMCJ" and aboriginal "Kuru" [20]: It is a rare neurodegenerative disease, which leads to a form of fatal progressive dementia. Clinical syndrome is characterized by mainly cortical polysexual deficits with memory loss, personality changes, hallucinations, dysarthria, myoclonus, postural rigidity and convulsions. CreutzfeldtJakob disease is the most frequent form of human spongiform encephalopathy. The prognosis is usually less than 2 years.
Normotensive hydrocephalus [21,22]: Means a type of communicating hydrocephalus, in which the increase in intracranial pressure, due to the accumulation of the cephalorachidian fluid, becomes stable or the formation of cerebrospinal fluid is balanced with absorption; intracranial pressure gradually decreases, but still remains at a slightly elevated level. Due to this balance the patient does not show the classic symptoms of intracranial hypertension such as headache, nausea, vomiting or unconsciousness. However, the subject shows the classic triad: difficult gait, urinary incontinence and mental decay. The prognosis is variable and depends on the surgery.

\section{Psychological symptoms}

Violent acts are not uncommon in patients with dementia, which are the main reason for the request for institutionalization. Disturbing actions include wandering, restlessness, shouting, throwing objects, physical violence, refusing treatment, incessant questions, hindering the work of staff members, insomnia and crying. Behavioral disturbances in dementia have not yet been well characterized in etiopathogenetic terms and the modality for their treatment is not fully clarified. Deciding which action constitutes a behavioral disorder is highly subjective. Tolerability (which actions the person assisting the patient manages to accept) also depends in part on the organization of the environment in which the patients live and in particular those relating to safety. For example, wandering can be tolerable if a patient lives in a safe environment (with locks and alarms on all doors and gates); however, if the patient is in an institution or hospital, wandering can be unmanageable since it disturbs other patients or interferes with staff activities. Many behaviors (for example, wandering, asking questions repeatedly, being uncooperative) are better tolerated during the day. It is not clear whether the sundowning (exacerbation of disturbing behavior at sunset or in the early hours of the evening) is due to a question of reduction of tolerability by the person assisting the patient or if it is a true diurnal variation [23].

Behavioral and psychological disorders can result from functional changes related to dementia: [1]

a) reduced inhibition of inappropriate behavior (for example, patients can undress in public places);

b) misinterpretation of visual and auditory signals (for example, they can resist treatments, which they perceive as aggression);

c) deterioration of short-term memory (for example, asking repeatedly for things that have already been answered);

d) reduced ability or inability to express needs (for example, they go around because they are alone, scared, or looking for something or someone).

The best approach is to characterize and classify behavior, rather than labeling all of these behaviors as agitation, a term with too many meanings to be useful. The Cohen-Mansfield shaking ladder is commonly used; classify behaviors as follows: [1] 
a) Physically aggressive: for example, hitting, pushing, kicking, biting, scratching, or grasping people or things.

b) Physically non-aggressive: for example, handling objects inappropriately, hiding things, dressing or undressing inappropriately, walking, repeating mannerisms or phrases, being restless, or trying to go elsewhere.

c) Verbally aggressive: for example, cursing, making strange noises, screaming, or with outbursts of anger.

d) Verbally non-aggressive: for example, complaining, whining, constantly asking for attention, not taking pleasure in anything, breaking in with relevant or irrelevant comments, being negative or domineering.

If behavioral variation is observed, an objective examination must be performed to rule out organic ailments and physical abuse, but also environmental modifications (for example, a person assisting the patient) must be considered, which may themselves be the cause of the disorder rather than a factor related to the patient. Depression, frequent among patients with dementia, can influence behavior and therefore must be identified. It may initially manifest as a sharp change in cognition, decreased appetite, mood changes, changes in sleep rhythm (often hypersomnolence), withdrawal, decreased level of activity, crying spells, death talk, sudden irritability or psychosis, or other sudden changes in behavior. Often, depression is first observed and detected by family members. Psychotic behavior must be identified because its management is different. The presence of delusions or hallucinations indicates psychosis. Delusions or hallucinations must be distinguished from disorientation, states of distress and misunderstandings that are frequent in patients with dementia: Delusions without paranoia can be confused with disorientation but the delusional content is usually fixed (for example, a medical residence is repeatedly called prison) while in disorientation it is variable (for example, a sanitary residence is sometimes called prison, then restaurant or house). Hallucinations occur without external sensory stimuli; hallucinations must be distinguished from illusions, which occur by misunderstanding external sensory stimuli (eg, cell phones, pagers) [24-28].

It is therefore evident that the best theoretical approach is determined by the perfect combination of medical therapy and supportive psychotherapy, to accompany the patient in the chronic, progressive and disabling evolution of the pathology. In particular, cognitive-behavioral and strategic therapy are more effective, together with drug therapy that acts on cognitiveness (for example, cholinesterase inhibitors), helping to better manage behavioral and psychological symptoms in patients with dementia. However, drugs that act predominantly on behavior (for example, antipsychotics) are used only when other approaches are ineffective and when they are critical to safety. The need for continuous treatment needs to be reviewed at least every month. Medicines must be selected to reduce the most intolerable behaviors. Antidepressants, preferably serotonin reuptake inhibitors, should only be prescribed for patients with obvious signs of depression. Antipsychotics are often used although their effectiveness has only been shown in psychotic patients. Other patients are unlikely to benefit from it and are likely to experience adverse effects, particularly the appearance of extrapyramidal symptoms. Tardive dyskinesias or tardive dystonia may develop; they often do not disappear with dose reduction or drug withdrawal. The choice of an antipsychotic is based on its relative toxicity. Among conventional antipsychotics, haloperidol is relatively low sedative and has less anticholinergic effects but is more likely to cause extrapyramidal symptoms; thioridazine and thixixene are less likely to cause extrapyramidal symptoms but are more sedative and have greater anticholinergic activity than haloperidol. Second generation (atypical) antipsychotics (for example aripiprazole, olanzapine, quetiapine, risperidone) have a weak anticholinergic action and produce fewer extrapyramidal side effects compared to conventional antipsychotics; however, these drugs, when used for an extended period, may be associated with an increased risk of hyperglycaemia and mortality from any cause. In addition, they can increase the risk of stroke in elderly patients who have dementia-related psychosis. If used, antipsychotics must be administered in low doses and for a short time, while antiepileptics, in particular valproate, can be useful in the control of sudden attacks of aggression. Finally, sedatives (for example, short-acting benzodiazepines) are sometimes used for a short period of time to relieve anxiety related to specific events but are not recommended for long-term therapy $[29,30]$.

\section{The Diagnostic Profiles}

As previously mentioned, the detailed anamnesis, serological laboratory tests, physical and neurological tests, electromyography, electroencephalogram and MRI or PET represent the first and second level diagnostic tests in the hypotheses of suspected dementia (genetic tests to be performed, which represent the third level of investigation). The clinical picture is completed by the psychological interview and the administration of a battery of neuropsychological tests calibrated on the patient (for example, the Mini-Mental Status Examination or the Montreal Cognitive Assessment), to best facilitate the exact diagnosis. In terms of dementia and impaired cognitive, for example, it is essential that the symptomatology involves two or more specific domains: a) impaired ability to acquire and remember new information (for example, to ask repetitive questions, often to put objects that are not in their place or to forget appointments); b) impaired reasoning and management of complex activities and poor judgment (for example, being unable to manage a bank account, making poor financial decisions); c) language dysfunction (for example, difficulty thinking common words, pronunciation and/or writing errors); d) visuospatial dysfunction (for example, the inability to recognize common faces or objects); e) changes in personality, attitude, or behavior [1].

\section{Conclusion}

Medical science is still a long way from discovering the pharmacological and genetic mechanisms that can block these pathologies and the challenge for the future will be to direct research in this direction. We still die of these diseases and the quality of 
life, especially in the last years of life, is strongly compressed in the negative. It must be a common commitment of all neuroscientific researchers to find solutions that are able to stem these morbid conditions, which destroy not only the patient but also the family members and those who care for them.

\section{References}

1. Perrotta G (2019) Psicologia clinica. Luxco.

2. Perrotta G (2019) Alzheimer's disease: Definition, contexts, neural correlates, strategies and clinical approaches. J Aging Stud Ther 1(1).

3. Mckee AC (2018) The neuropathology of chronic traumatic encephalopathy. Handb Clin Neurol 158: 297-307.

4. Sivasathiaseelan H, Marshall CR, Agustus JL, Benhamou E, Bond RL, et al. (2019) Frontotemporal dementia: A clinical review. Semin Neurol 39(2): 251-263.

5. Marshall CR, Hardy CJD, Volkmer A, Russell LL, Bond RL, et al. (2018) Primary progressive aphasia: A clinical approach. J Neurol 265(6): $1474-1490$.

6. Klimova B, Novotny M, Kuca K (2018) Semantic dementia: A mini review. Mini Rev Med Chem 18(1): 3-8.

7. Armstrong MJ (2018) Progressive supranuclear palsy: An update. Curr Neurol Neurosci Rep 18(3): 12.

8. Sanford AM (2018) Lewy body dementia. Clin Geriatr Med 34(4): 603615.

9. Perrotta G (2019) Parkinson's disorder: definition, contexts, neural correlates, strategies and clinical approaches. Journal of Neuroscience and Neurological Surgery 4(5).

10. Newhouse A, Chemali Z (2019) Neuroendocrine disturbances in neurodegenerative disorders: A scoping review. Psychosomatics 61(2): 105-115.

11. Rosenberg GA (2018) Binswanger's disease: Biomarkers in the inflammatory form of vascular cognitive impairment and dementia. Neurochem 144(5): 634-643.

12. Regenhardt RW, Das AS, Lo EH, Caplan LR (2019) Advances in understanding the pathophysiology of lacunar stroke: A Review. JAMA Neurol 75(10): 1273-1281.

13. Regenhardt RW, Das AS, Ohtomo R, Lo EH, Ayata C, et al. (2019) Pathophysiology of lacunar stroke: History's mysteries and modern interpretations. J Stroke Cerebrovasc Dis 28(8): 2079-2097.

14. Kalaria RN (2018) The pathology and pathophysiology of vascular dementia. Neuropharmacology 134(Pt B): 226-239.
15. Brien JT, Thomas A (2015) Vascular dementia. Lancet 386(10004): 1698-1706.

16. Park YH, Kang MJ, Suh J, An SS, Kim S (2020) Pearls \& Oy-sters challenging diagnosis of gerstmann-sträussler-scheinker disease: clinical and imaging findings. Neurology 92(2):101-103.

17. Cracco L, Appleby BS, Gambetti P (2018) Fatal familial insomnia and sporadic fatal insomnia. Handb Clin Neurol 153: 271-299.

18. Llorens F, Zarranz JJ, Fischer A, Zerr I, Ferrer I (2017) Fatal familial insomnia: Clinical aspects and molecular alterations. Curr Neurol Neurosci Rep 17(4): 30.

19. Damavandi PT, Dove MT, Pickersgill RW (2017) A review of drug therapy for sporadic fatal insomnia. Prion 11(5): 293-299.

20. Narula R (2018) Creutzfeldt-jakob disease. N Engl J Med 378(4): e7.

21. Kolecka M, Farke D, Failling K, Kramer M, Schmidt MJ (2019) Intraoperative measurement of intraventricular pressure in dogs with communicating internal hydrocephalus. PLoS One 14(9): e0222725.

22. Barba G, Barbera C, Brazzarola M, Marangoni S (2016) Recovery from confabulation after normotensive hydrocephalus shunting. Cortex 75: 82-86.

23. Perrotta G (2019) Psicologia generale. Luxco.

24. Perrotta G (2019) Anxiety disorders: definitions, contexts, neural correlates and strategic therapy. Journal of Neurology and Neuroscience 6(1): 046.

25. Perrotta G (2019) Sleep-wake disorders: Definition, contexts and neural correlations. J Neurol Psychol 7(1): 09.

26. Perrotta G (2019) Delusions, paranoia and hallucinations: definitions, differences, clinical contexts and therapeutic approaches. Cientific Journal of Neurology 1(1): 8-14.

27. Perrotta G (2019) The reality plan and the subjective construction of one's perception: The strategic theoretical model among, sensations, perceptions, defence mechanisms, needs, personal constructs, beliefs system, social influences and systematic errors. J Clinical Research and Reports 1(1).

28. Perrotta G (2019) Psychological trauma: definition, clinical contexts, neural correlations and therapeutic approaches recent discoveries. Curr Res Psychiatry Brain Disord: CRPBD-100006.

29. Lochhead JD, Michele AN, Gerald AM (2016) The treatment of behavioral disturbances and psychosis associated with dementia. Psychiatr Pol 50(2): 311-322.

30. Perng CH (2018) The treatment of cognitive dysfunction in dementia: A multiple treatments meta-analysis. Psychopharmacology 235(5): 15711580. 